\title{
Quantification of PLA Contamination in PET during Injection Moulding by In-line NIR Spectroscopy
}

Andrew R McLauchlin ${ }^{a}$ (Corresponding author); Oana Ghita ${ }^{a}$; Ali Gahkani ${ }^{b}$

a College of Engineering Mathematics and Physical Sciences, Harrison Building, University of Exeter, North Park Road, Exeter EX4 4QF

b Bruker UK Limited, Banner La, Coventry CV4 9GH

Corresponding Author:

Andrew R McLauchlin a.mclauchlin@exeter.ac.uk

College of Engineering Mathematics and Physical Sciences, Harrison Building, University of Exeter, North Park Road, Exeter EX4 4QF

+44 (0)1392 722603 


\section{Abstract}

In-line spectroscopy is a useful tool for monitoring processing conditions and polymer quality in the melt state in processes such as extrusion and injection moulding. This study extends the scope of this technique to the quantitative detection of poly(lactic acid) (PLA), which is a contaminant of concern in recycled poly(ethylene terephthalate) (PET). The near-infrared spectra of thermally treated PLA were studied for evidence of spectral changes which could be used as markers for PLA in PET, and these are discussed in detail. Several chemometric models for quantifying PLA in PET were developed and the influence of chemical or physical changes on the performance of the models is discussed. The selection of the final model was based on criteria aimed at eliminating interference due to spectral changes resulting from physical processes to give a robust model based specifically on the chemistry of the polymers studied. Finally, the models were tested against a new test mixture of known composition and the outputs are discussed.

Keywords

In-line spectroscopy; NIR; chemometric; recycling; injection moulding; PLA 


\section{Introduction}

The European Packaging and Waste Directive (94/62/EC), revised in 2004 to (2004/12/EC), required that between 55 and $80 \%$ by weight of packaging waste be recycled by December 2008 and that the minimum proportion of plastic waste be $22.5 \%$. In the case of poly(ethylene terephthalate) (PET) this latter target has driven the reprocessing of recycled PET ( $r P E T$ ) into food grade material, where its price can match that of virgin PET. Food grade rPET must conform to criteria set out in Commission Regulation EC 282/2008, which means that recyclers need to reduce all contaminants to below prescribed levels. Despite constant advances in plastics sorting technology, there remain several threats to the quality of $r$ PET. One such threat is from bioplastics such as poly(lactic acid) (PLA) which represent a relatively new type of thermoplastic material derived from renewable biomass sources. Driven by the industry need to develop bio-based materials to replace the petroleum based plastics, production of PLA has been estimated to expand from f6.6billion to f10billion of PLA annually [1].

PLA has received particular attention and divided opinion where recycling of packaging is concerned. PLA can be used to make drinks bottles which to the consumer appear to be the same as PET bottles, and PLA bottles are often put into plastic bottle recycling bins as a result. However the two polymers are incompatible and even small amounts of PLA in PET can have deleterious effects on its rheological properties, although negative effects on mechanical properties are only observed in certain conditions [2]. Furthermore, PLA, which melts at $180^{\circ} \mathrm{C}$, may degrade at the processing temperature of PET $\left(>260^{\circ} \mathrm{C}\right)$, so that even a small amount of PLA, i.e. $<0.1 \%$, results in clouded PET bottles, thereby spoiling the batch. The immiscibility of PET and PLA has been demonstrated by scanning electron microscopy [2] which revealed a biphasic morphology that explains the cloudiness of PET contaminated by PLA.

The production of PLA is increasing annually and, with more drinks manufacturers adopting PLA, the threat of contamination of PET recyclate is ever-present. Although sorting technologies are now capable of eliminating almost all PLA bottles, the potential for contamination still exists and PET recyclers wish to eliminate all PLA contamination with $100 \%$ confidence so as to ensure consistent product quality.

The need for understanding material performance in processing, either moisture absorption, colour mixing or percentage of co-polymers is constantly coming up as a necessity for good quality manufacturing and subsequent reduction of manufacturing waste. NIR spectroscopy has been successfully applied in the past to investigate material properties during in-line injection moulding and extrusion [3-8]. Relying on the sensitivity of the NIR system to detect material chemical and physical changes, NIR spectroscopy has also been used to assess the dispersion of fillers and nanoparticles in polymers during compounding $[9,10]$. In all cases, the technique proved capable of providing very good 
results qualitatively and quantitatively. However, the technique should be used with care, as a previous study [11] showed that machine parameters, such as nozzle temperature and injection moulding speed, can significantly influence the material physical parameters and complicate the spectral analysis. It is, therefore, important that processing parameters be kept constant when developing an in-line analytical method.

To date, only one previous study investigated the separation of PET and PLA [12]. The authors used a hyperspectral imaging (HSI) technique to discriminate between the two polymers. This method combined the spatial information characteristic of imaging techniques and the spectral details. Spectral data was recorded from every pixel across the image and it was suggested that this provided a more comprehensive measure of the chemical composition as derived from spectral data. Several chemometric methods: Principle Component Analysis (PCA); Partial Least Squares-Discriminant Analysis (PLS-DA) and Multivariate Curve Resolution (MCR) were used to analyse the collected data. The PLSDA model identified the region $1600-1650 \mathrm{~nm}\left(6250-6060 \mathrm{~cm}^{-1}\right)$, corresponding to first overtone of the $\mathrm{C}-\mathrm{H}$ stretching vibration, as the most significant one. This is in agreement with the current study, which also identified the $\mathrm{C}-\mathrm{H}$ stretching region as being significant for the model prediction. However, in order to eliminate any further errors derived from baseline shifts the second overtone of the $\mathrm{C}-\mathrm{H}$ stretching region, $9000-8000 \mathrm{~cm}^{-1}$, has been considered as most appropriate here.

NIR has been used extensively as an in-line technique for other applications such as process control; consequently there are many turnkey NIR-based systems in this industrial sector. However, its use in plastics waste sorting remains unique and important due to the increasing market value and overall life cycle of these materials. While HSI systems will undoubtedly become a major component of future sorting systems as the demand grows for sorting systems capable of processing increasingly complex recyclate streams, there is scope for the development of a system based on existing NIR technology which can be easily redeployed in the injection nozzle and further linked to end of life separation .

This study presents an investigation into the sensitivity and accuracy of the in-line NIR system of monitoring the presence of PLA traces in PET during PET processing when PLA elimination is not $100 \%$ achieved. The study discusses the influence of the selected spectral regions and spectral processing parameters on the quality of the proposed models.

\section{Materials \& Methods}

\subsection{Material Preparation}

Melinar Laser+ injection grade PET was obtained from Distrupol Limited, UK. Injection moulding grade PLA was obtained from Bioresins.eu (Olney, Bucks MK46 5FP UK). 
PLA pellets were converted to a fine powder $(<0.2 \mathrm{~mm}$ particle size) by cryogenic grinding using liquid nitrogen as the coolant. All materials were dried at $107^{\circ} \mathrm{C}$ for 8 hours prior to processing trials. Cryogenically ground PLA was, therefore, subjected to the same conditions as the PET throughout.

\subsection{Injection moulding and In-line Spectroscopy}

Samples were prepared by injection moulding using a Battenfeld HM40 4/130 Injection Moulder with the conditions listed in Table 1 . Blends of PET containing PLA in the range $0.01 \%$ to $0.09 \% \mathrm{w} / \mathrm{w}$, in $0.01 \%$ increments, were prepared by pre-blending the dried resin pellets and powdered PLA in a container before adding to the feed hopper of the injection moulder. The concentration of the PLA in PET was calculated from the known weight of each component in the mixture. Near infrared spectra of the polymer melt were acquired by using an injection nozzle adapted to accommodate two fibre optic probes (FOS Messtechnik, Germany) aligned so that the beam was perpendicular to the polymer melt flow, as shown in Figure 1 and previous studies [6,11].

For in-line measurements, the two optical fibre probes attached to the injection-moulding machine were connected to a Bruker Matrix process FT-NIR Spectrometer [6]. The FT-NIR spectrometer operated in the near infrared region from $4000-12000 \mathrm{~cm}^{-1}$, transmittance mode, using a white light source and a TE-InGaAs detector, along with a quartz beam splitter. The path length, i.e. distance between the probe windows was $8 \mathrm{~mm}$. The spectra were collected during the hold phase of the injection moulding cycle, when the holding pressure, i.e. the pressure in the nozzle immediately after injection and prior to refilling the barrel, was set to be constant. This holding phase lasted six seconds, which was long enough to carry out 10 scans at $4 \mathrm{~cm}^{-1}$ resolution. Thus, a spectrum was collected for each injection moulding cycle from the time the hopper was charged with the PLA/PET mix until 100 spectra had been collected. The calibration curves were compiled using the $61^{\text {st }}$ to the $80^{\text {th }}$ spectrum (i.e. 20 spectra) for each concentration of PLA as it was known from previous experiments that these spectra showed a good degree of consistency.

\subsection{Preparation of Heat Treated PLA}

In order to obtain reference materials representative of PLA subjected to the thermal environment of injection moulding conditions, samples of injection grade PLA $(0.030 \mathrm{~g})$ were placed in deep aluminium crucibles (100ul capacity) and heated to $265^{\circ} \mathrm{C}$ under nitrogen and held at that temperature for $0,1,2,5,10,15$ and 20 minutes. After heat treatment, the cooled PLA discs were removed from the aluminium crucibles for spectroscopic measurement.

Near-infrared reflectance spectra of the heat treated PLA discs were collected using a QR400-7-VIS-NIR reflectance probe (Ocean Optics) connected to the Bruker Matrix spectrometer used for the in-line NIR measurements. Spectra for each sample were recorded by averaging 32 scans at $4 \mathrm{~cm}^{-1}$ resolution. These spectra were used to identify 
spectral regions which were related to PLA rather than PET and which could be used in the chemometric modelling.

\subsection{Chemometric Modelling}

The spectral dataset was prepared for multivariate analysis by dividing the spectra into subregions as shown in Table 2 . To produce model $A$, the spectra were divided into five regions which represented the following categories: $\left(1200-7500 \mathrm{~cm}^{-1}\right) 3^{\text {rd }}$ and $2^{\text {nd }}$ overtones of $\mathrm{C}-\mathrm{H}$ bonds; $\left(7500-6100 \mathrm{~cm}^{-1}\right) 1^{\text {st }}$ overtones of O-H groups; $\left(6100-5450 \mathrm{~cm}^{-1}\right) 1^{\text {st }}$ overtones of $\mathrm{C}-\mathrm{H}$ bonds; $\left(5450-4805 \mathrm{~cm}^{-1}\right) 2^{\text {nd }}$ overtones of $\mathrm{C}=\mathrm{O}$ groups together with $\mathrm{O}-\mathrm{H}$ combination bands; $\left(4805-4250 \mathrm{~cm}^{-1}\right)$ combinations of $\mathrm{C}-\mathrm{H}$ bonds. In this particular case, these were the default regions preselected by the OPUS software. For models $B, C, D, E$ and $F$, spectral regions identified as being specific to PLA were selected by inspection of the NIR spectra of the thermally treated PLA samples: these are also listed in Table 2.

To produce multivariate regression models, the data was subjected to Partial Least Squares (PLS) analysis. In contrast to univariate regression in which one factor, such as absorbance at a single wavelength, is used to predict the concentration of a component of a mixture, this statistical technique produces regression models which use the least number of factors to relate as much of the variation in the spectra as possible to the property of interest, in this case PLA concentration. In the output of the analysis, the number of factors is given by the 'rank', which is the number of independent sources of variation that correlate with the property of interest as well as spectral variance. A comprehensive explanation in the context of chemometric analysis is given by Martens and Naes [13].

All the models were generated by the PLS-1 method using cross-validation, with one spectrum being left out for the validation step, so that each spectrum in turn was tested against the model. The spectra were mean-centred prior to analysis. Two optimisation algorithms for model generation were used, both of which are embodied in the spectral software. The "NIR" algorithm tested each spectral sub-region individually as well as in all possible permutations: this method was used to produce model " $A$ ". The "GenB" algorithm first identified the sub-region giving the lowest Root Mean Square Error of Cross validation (RMSECV). The remaining sub-regions were similarly tested in turn to find the best one to add to the first. This was repeated until there is no significant decrease in the RMSECV. Finally, the best combination of sub-regions was found by adding or removing further subregions until there was no improvement. The optimisations were run with the following spectral preprocessing methods selected: no preprocessing; straight line subtraction; minmax normalisation; first derivative and second derivative. Models were selected from the generated output which had low values of RMSECV and whose validation plots indicated good correspondence between predicted and true values. The calibration plots were not used for selection purposes as these would have given an optimistic view of the model.

Model A was selected from the output of the optimisations using the NIR procedure and was used to evaluate a model based on no spectral preprocessing, using default general 
spectral regions, as explained above. Models B to F were produced by the GenB procedure, which is more thorough in that it tests more permutations of spectral regions than the NIR procedure. Model B was selected as an example of a model with no spectral preprocessing which used PLA-specific spectral regions and could be compared with model A. Model C was derived from model $B$ in order to test the significance of the spectral region 9000$8240 \mathrm{~cm}^{-1}$ by removing this region and revalidating the model.

To remove the effects of baseline shift, models $D-F$ were based on first derivative preprocessing of the spectra. Model D used the same PLA specific spectral regions as model $B$, and thus enabled the effect of first derivative processing to be clearly seen; model $E$ tested the spectral region $9000-8240 \mathrm{~cm}^{-1}$ in isolation and, finally, model $\mathrm{F}$ tested the effect of adding the spectral region $5400-4650 \mathrm{~cm}^{-1}$ to model $\mathrm{E}$. The input parameters and output performance criteria for all the models are shown in Table 3.

\section{Results and Discussion}

The NIR spectra of PLA pellets subjected to thermal treatments are presented in Figure 2 and Figure 3. No major differences between spectra recorded at different temperatures and times were noticed in the NIR region. The variation in intensity is related to the sample morphology and physical changes recorded when collecting the spectra, and no obvious structural changes were noticed. If the near infrared reflectance spectra of PET and PLA are compared, as in Figure 4, there is an obvious difference between them in the region 9000$8000 \mathrm{~cm}^{-1}$, which is the region corresponding to $2^{\text {nd }}$ overtones of $\mathrm{C}-\mathrm{H}$ stretching vibrations. In this region, PET shows a peak at $8845 \mathrm{~cm}^{-1}$ while PLA shows a peak at $8794 \mathrm{~cm}^{-1}$. There is, therefore, potential for distinguishing these two materials using this region of the NIR spectrum. A peak at $5251 \mathrm{~cm}^{-1}$ in PET pellet shifts to $5116 \mathrm{~cm}^{-1}$ in the polymer melt and is attributed to the $2^{\text {nd }}$ overtone of the ester $C=O$ stretch: this peak is, therefore, not a unique marker for PLA as the ester group is present in both PET and PLA.

NIR spectra of the PET polymer collected by the in-line spectroscopy system showed no peak changes but clear physical changes were noticed through the baseline (Figure 5) where the increase in concentration of PLA in PET led to a systematic baseline shift. Higher concentrations of PLA resulted in increasing opacity of the polymer melt, which led to a decrease in light intensity and, therefore, a baseline shift. Previous studies [11] suggested that this shift could be used as a method to monitor the changes in colour or additive concentration when no chemical changes are taking place. Depending on the source of the physical changes, attention should be given to whether chemical and physical changes should both be considered when building calibration models. 
The emergence of the PLA/PET mixture from the injection moulding nozzle over time is shown in figure 6 , which is a plot of the conformity index $(\mathrm{Cl})$ against spectrum number for the trial conducted with $0.09 \%$ PLA. The conformity index was calculated for the unprocessed spectra by calculations given elsewhere [11]. It can be clearly seen that the emergence of the new mixture is detected after about twenty shots and that the conformity index increases until it reaches a steady state, at which point the spectra can be considered to be representative of the test mixture and can be used for the chemometric modelling. The plot also shows how sensitive the in-line spectroscopy system is to small changes in the composition of the polymer melt, as all other conditions, such as temperature, hold time and injection pressure were kept constant during the injection moulding run.

Figure 7 shows the cross-validation plots for the chemometric models (a)A, (b)B, (c)C and (d)E and the output parameters are given in Table 3, which lists the method of spectral region selection (software default or PLA specific); the optimisation algorithm (NIR or GenB); the spectral preprocessing (none or $1^{\text {st }}$ derivative); the rank (equivalent to the number of factors used in the PLS); the Root-Mean Square Error of Covariance (RMSECV) and the coefficient of determination $\left(r^{2}\right)$ which approaches $100 \%$ as the predicted concentrations approach the true values. The validation plots are shown in preference to the calibration plots because the latter show the fitted values for PLA\% and, as a result, the calibration plot tends to suggest a good fit, whereas the cross-validation plot is a more stringent visual representation of the fit of the predicted values. Thus, the cross-validation plot for model $A$ shows good correspondence between predicted and true values for PLA\% in PET, and this is supported by an $r^{2}$ value of $98.2 \%$ and a REMSECV value of 0.0035 , both of which indicate a low error in prediction. This model, which was generated using the NIR algorithm of the Quant2 tool, used predefined spectral regions which are not necessarily specific to the spectrum of PLA (Table 3). The region $9750-7500 \mathrm{~cm}^{-1}$ contains the second overtone vibrations of $\mathrm{C}-\mathrm{H}$ stretches as well as first overtone vibrations of $\mathrm{C}-\mathrm{H}$ combination bands, while the region $5450-4600 \mathrm{~cm}^{-1}$ contains the second overtones of carbonyl stretches as well as first overtones of $\mathrm{O}-\mathrm{H}$ stretches, including those due to water, although this was not expected to be present as all materials were dried prior to running the injection moulding trials.

Replacing the default regions selected by the NIR algorithm with spectral regions that encompass strong absorbances by PLA gave model $B$, whose validation curve can be seen in Figure $7 \mathrm{~b}$. This was visually very similar to that of model $A$ and also had similar RMSECV and $r^{2}$ values. If the spectral regions of models $A$ and $B$ are compared, the region $9750-7500 \mathrm{~cm}^{-1}$ in model $A$ has been significantly narrowed to $9000-8240 \mathrm{~cm}^{-1}$ in model $B$, while the region $5450-4600 \mathrm{~cm}^{-1}$ has been slightly narrowed to $5400-4650 \mathrm{~cm}^{-1}$. An extra region, $6711-$ $5900 \mathrm{~cm}^{-1}$, was introduced in model $\mathrm{B}$ : while this region includes the $1^{\text {st }}$ overtone of $\mathrm{C}-\mathrm{H}$ stretches of the methyl $\left(-\mathrm{CH}_{3}\right)$ groups, it can also include $1^{\text {st }}$ overtone bands from aromatic $\mathrm{C}-\mathrm{H}$ stretches and, therefore, is at risk of being swamped by signals from the PET, which is an aromatic polyester. This region could, therefore, be modelling PET rather than 
information about the minority component, PLA. The spectra of PET and PLA show some important differences in the region $9000-8240 \mathrm{~cm}^{-1}$ and when this region was removed from model $B$ and the dataset revalidated to give model $C$, it can be seen in Figure $7 c$ that this model is visually a poorer fit to the 1:1 target line and that the RMSECV has increased to 0.0094 , indicating increased error in prediction, while the $r^{2}$ value has decreased to $88.3 \%$, again indicating a poorer model and the optimal rank number has increased to 7 , making the model less stable.

Therefore, the region $9000-8240 \mathrm{~cm}^{-1}$ contained significant information for constructing an accurate and stable model. This was confirmed by selecting from the optimisation output a model (E) which used only the region $9000-8042 \mathrm{~cm}^{-1}$, and which applied first derivative preprocessing, which has the effect of removing information arising from any shift in the baseline, such as can be seen in the spectra in Figure 5. A principle component analysis of model $E$ showed that the first two factors explained $89.7 \%$ and $1.1 \%$ of the spectral variance, respectively, while the total spectral variance explained by the first 6 factors was $92.8 \%$.

The four models were tested for accuracy by applying spectra from a separate injection moulding run in which the PLA concentration was $0.04 \%$. In order to test the robustness of the chemometric models, this run was performed on a separate day to the calibration runs and on a completely new resin pellet mixture. As with the calibration spectra, twenty consecutive spectra from the steady state period of the run were used to test the four selected models. The predicted concentrations from the four models are shown in Figure 8. All three of the models that were based on no spectral preprocessing overestimated the concentration by an order of magnitude. The first model produced using the default spectral ranges $(A)$ gave predicted PLA concentrations around $0.3 \%$. Using spectral regions more specific to PLA (model $B$ ) resulted in higher predicted concentrations, while removing the spectral region $9000-8042 \mathrm{~cm}^{-1}$ gave still higher predicted values, attesting to the importance of this region. The best predictions were given by the model based on first derivative preprocessing of the spectral region $9000-8042 \mathrm{~cm}^{-1}$ (model E), which predicted concentrations that were closest to the target value of $0.04 \%$. It can be seen that the models demonstrated different sensitivities to spectral variations, especially the third and fourth spectra, which gave noticeably higher predicted values with models $A$ and $E$. If these two spectra are discounted, then the average value predicted by $E$ for the test dataset was $0.066 \% \pm 0.006 \%$ which, although an overestimation, is a considerable improvement over the models based on no preprocessing.

Finally, the importance of first derivative processing can be seen by comparing models $B, D$ and $E$. The output parameters, seen in Table 3, show a poorer fit $\left(r^{2}=70.03 \%\right)$, greater error in prediction (RMSECV $=0.0142$ ) and higher rank number (9) for model $D$ compared to $E$. Therefore the spectral regions $6711-5900 \mathrm{~cm}^{-1}$ and $5400-4650 \mathrm{~cm}^{-1}$ contribute to error when first derivative preprocessing is applied, as can be seen by comparing models $D, E$ and $F$. A 
further indication that these regions create instability in the model was shown by the mean predicted value of $0.109 \% \pm 0.022 \%$ that model $D$ gave for the test spectra.

\section{Conclusions}

PLA contamination in PET can be detected quantitatively in the polymer melt in the range $0.01 \%-0.09 \%$ by in-line near-infrared spectroscopy. Chemometric models can be made more specific to PLA by excluding the spectral region of wavenumber greater than $9000 \mathrm{~cm}^{-}$ ${ }^{1}$, which shows a systematic shift in baseline due to increasing turbidity in the processed material, and inclusion of which would render the model nonspecific and vulnerable to interference. This interference, in the form of baseline shift throughout the spectrum, can be mitigated by using first derivative preprocessing. Strategic selection of a spectral region wherein PET and PLA show clear differences gave rise to a more stable and accurate model compared to one based on default parameters. PLA concentrations can, therefore, be quantitatively estimated from changes in the region associated with second overtone carbon-hydrogen stretches to give a stable model with improved prediction over a simple baseline-dependent model.

\section{Acknowledgement}

This research was funded by Grant Agreement No. 285889 under the Seventh Framework Programme Theme SME-2011-2. 
[1] P.R. Gruber, Commodity polymers from renewable resources: Polylactic acid., in: Carbon Manag. Implic. R\&D Chem. Sci. Technol. Work. Rep. to Chem. Sci. Roundtable. Natl. Acad. Sci., National Center for Biotechnology Information, U.S Library of Medicine, 8600 Rockville Pike, Bethesda MD 20894 USA, Bethesda MD, 2001.

[2] F.P. La Mantia, L. Botta, M. Morreale, R. Scaffaro, Effect of small amounts of poly(lactic acid) on the recycling of poly(ethylene terephthalate) bottles, Polym. Degrad. Stab. 97 (2012) 21-24.

[3] H. Lammers, M.P.B. Van Uum, J.P. De Kleijn, Process analysis: properties of poly(ethylene terephthalate) measured by near infrared spectroscopy, 2. In-line analysis of poly(ethylene terephthalate) melt, Macromol. Chem. Phys. 196 (1995) 2029-2034.

[4] D. Fischer, J. Müller, S. Kummer, B. Kretzschmar, Real Time Monitoring of Morphologic and Mechanical Properties of Polymer Nanocomposites During Extrusion by near Infrared and Ultrasonic Spectroscopy, Macromol. Symp. 305 (2011) 10-17.

[5] T. Rohe, W. Becker, S. Kölle, N. Eisenreich, P. Eyerer, Near infrared (NIR) spectroscopy for in-line monitoring of polymer extrusion processes., Talanta. 50 (1999) 283-90.

[6] O. Dumitrescu, D. Baker, G. Foster, K. Evans, Near infrared spectroscopy for in-line monitoring during injection moulding, Polym. Test. 24 (2005) 367-375.

[7] P.D. Coates, S.E. Barnes, M.G. Sibley, E.C. Brown, H.G.M. Edwards, I.J. Scowen, Inprocess vibrational spectroscopy and ultrasound measurements in polymer melt extrusion, Polymer (Guildf). 44 (2003) 5937-5949.

[8] D. Fischer, T. Bayer, K.-J. Eichhorn, M. Otto, In-line process monitoring on polymer melts by NIR-spectroscopy, Fresenius. J. Anal. Chem. 359 (1997) 74-77.

[9] J.M. Barbas, A. V. Machado, J. a. Covas, In-line near-infrared spectroscopy: A tool to monitor the preparation of polymer-clay nanocomposites in extruders, J. Appl. Polym. Sci. (2012) n/a-n/a.

[10] A. Witschnigg, S. Laske, M. Kracalik, M. Feuchter, G. Pinter, G. Maier, et al., In-line characterization of polypropylene nanocomposites using FT-NIR, J. Appl. Polym. Sci. 117 (2010) 3047-3053.

[11] O.R. Ghita, D.C. Baker, K.E. Evans, An in-line near-infrared process control tool for monitoring the effects of speed, temperature, and polymer colour in injection moulding, Polym. Test. 27 (2008) 459-469. 
[12] A. Ulrici, S. Serranti, C. Ferrari, D. Cesare, G. Foca, G. Bonifazi, Efficient chemometric strategies for PET-PLA discrimination in recycling plants using hyperspectral imaging, Chemom. Intell. Lab. Syst. 122 (2013) 31-39.

[13] H. Martens, T. Naes, Multivariate Calibration, John Wiley \& Sons Ltd, Chichester, 1989. 
Figures

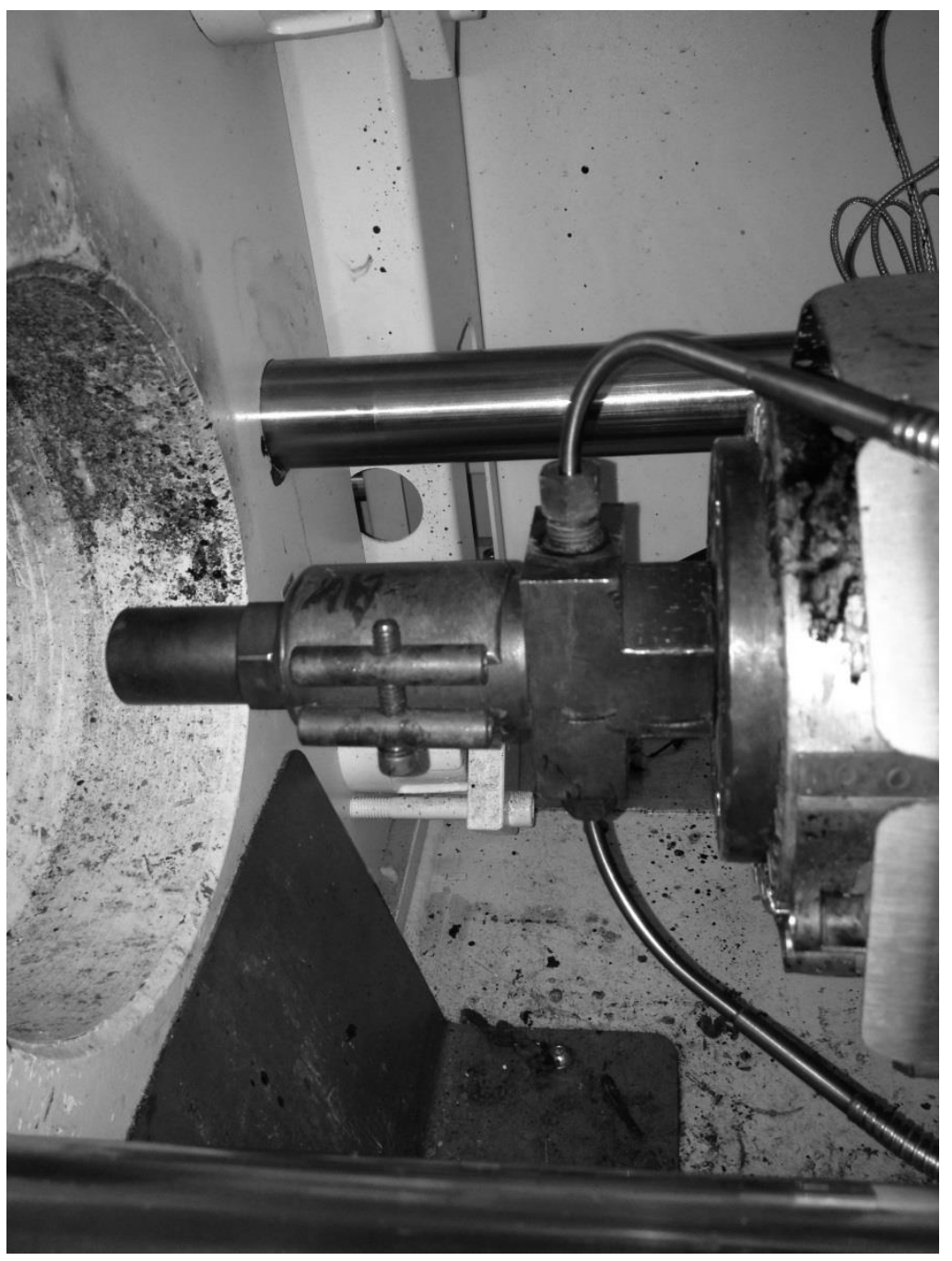

Figure 1. View of the modified injection nozzle with in-line NIR probes fitted to the top and bottom ports. 


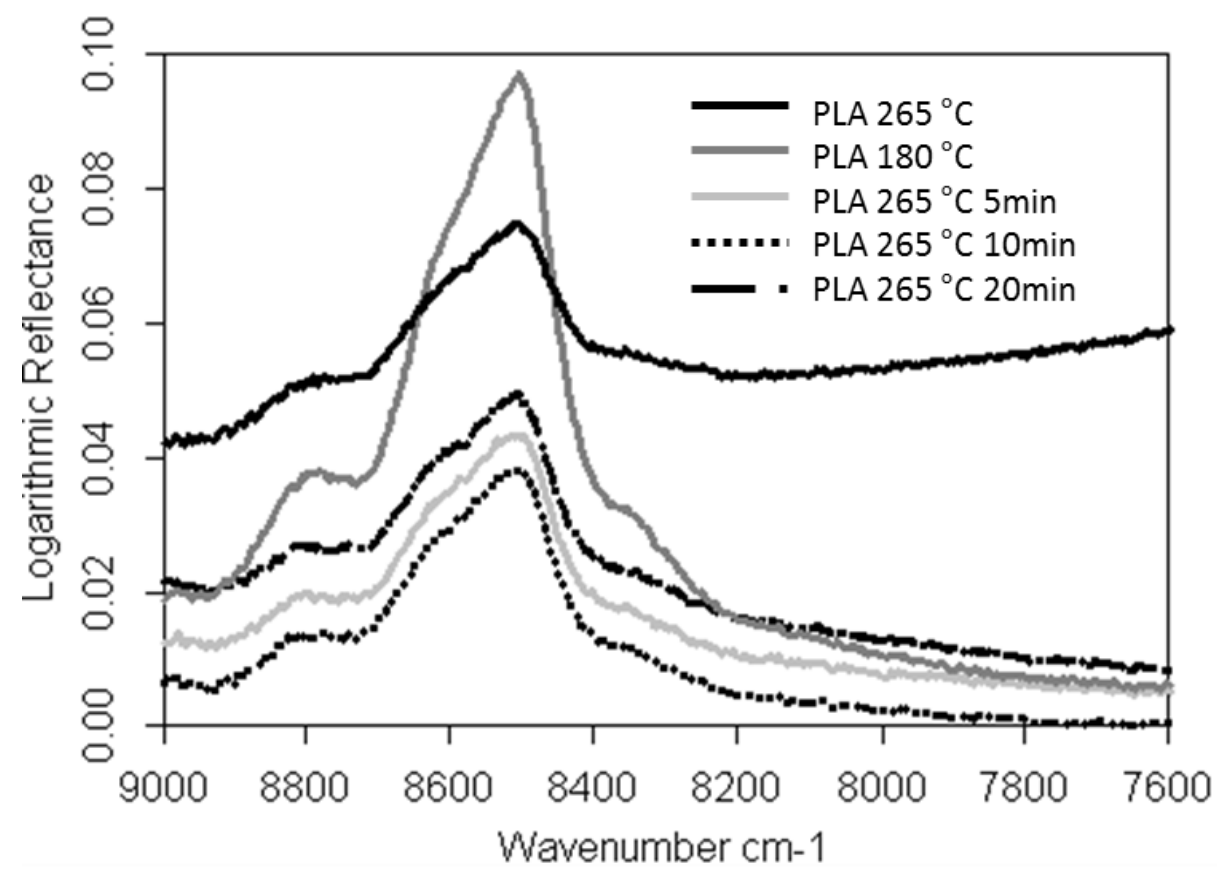

Figure 2. Near-infrared spectra of PLA pellet and heated PLA samples: expansion of region $9000-7600 \mathrm{~cm}^{-1}$.

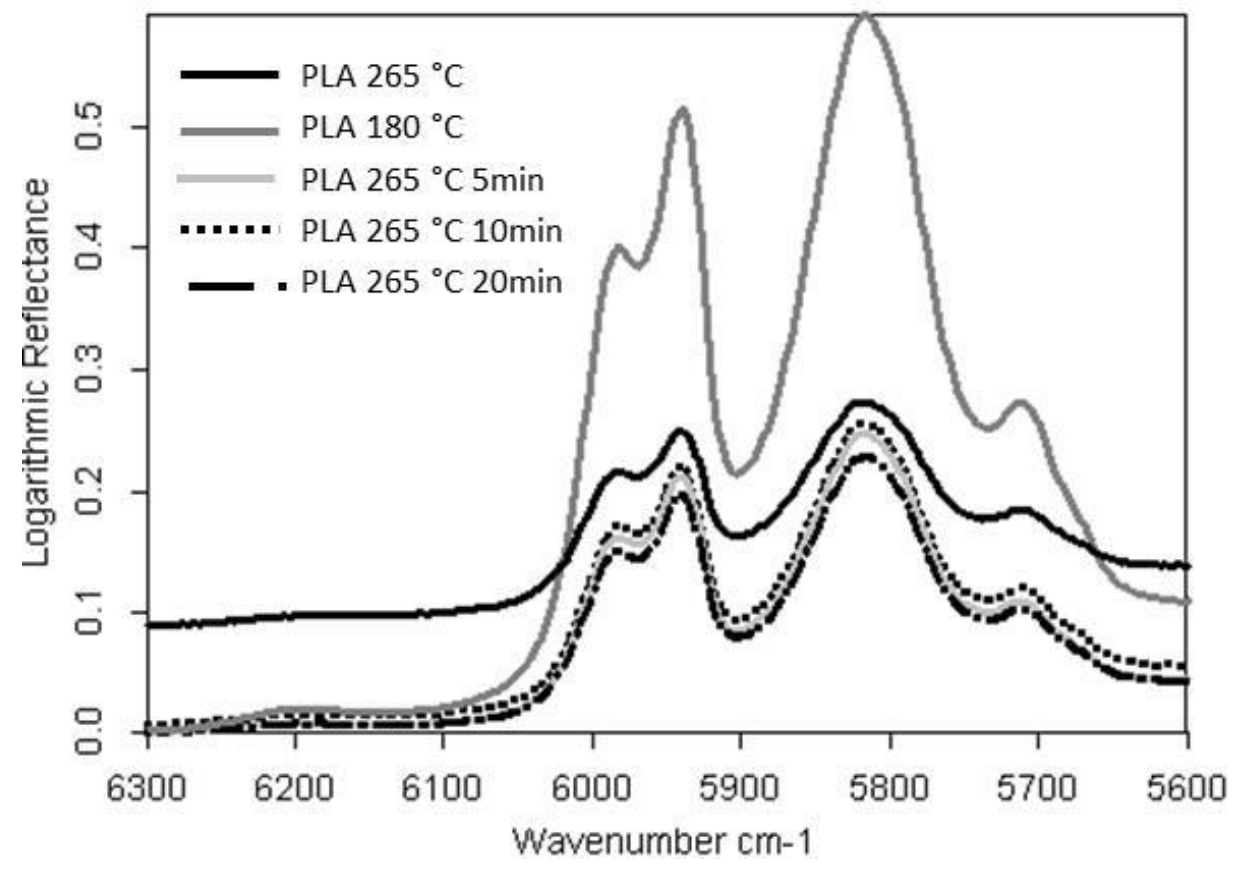

Figure 3. Near-infrared spectra of PLA pellet and heated PLA samples: expansion of region $6300-5600 \mathrm{~cm}^{-1}$. 


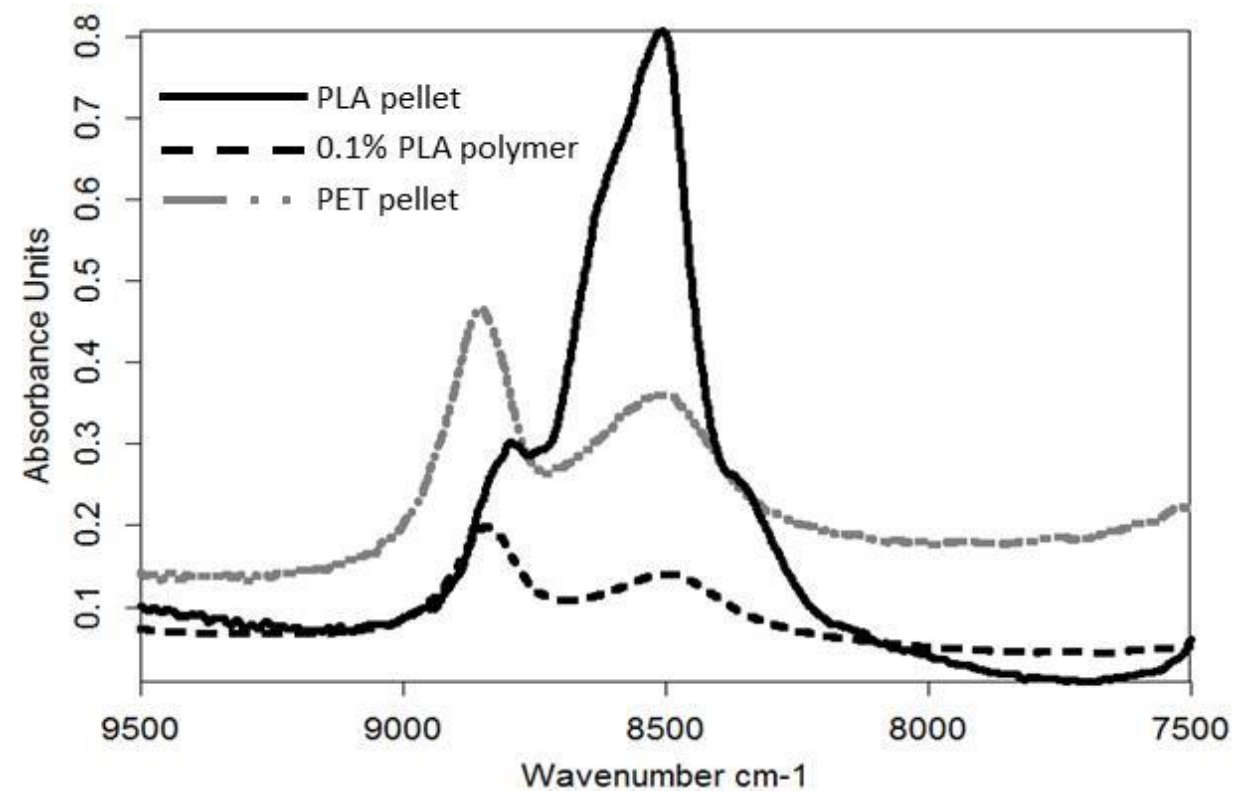

Figure 4. Near-infrared reflectance spectra of PLA pellets (top) and PET pellets (bottom).

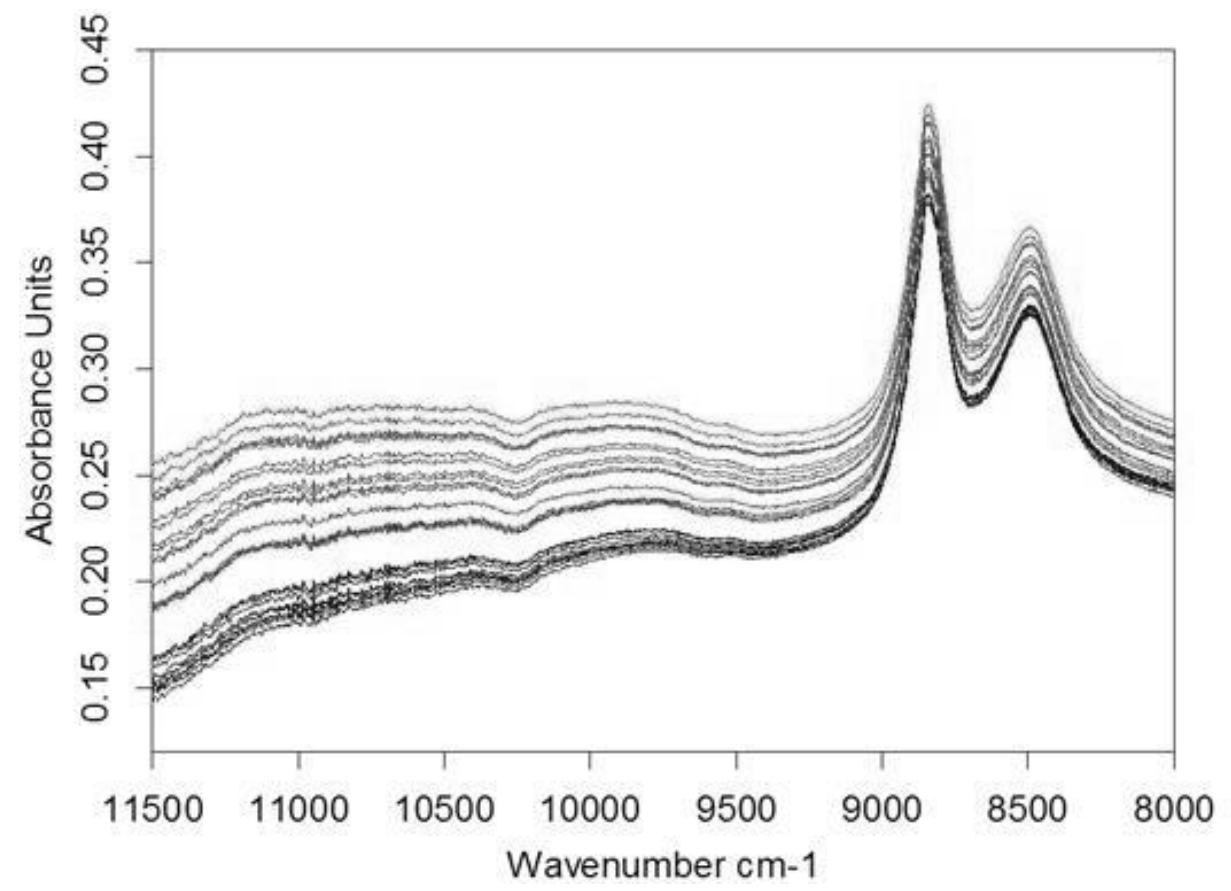

Figure 5. Absorbance spectra of PET/PLA polymer melts collected by in-line near-infrared spectroscopy. 


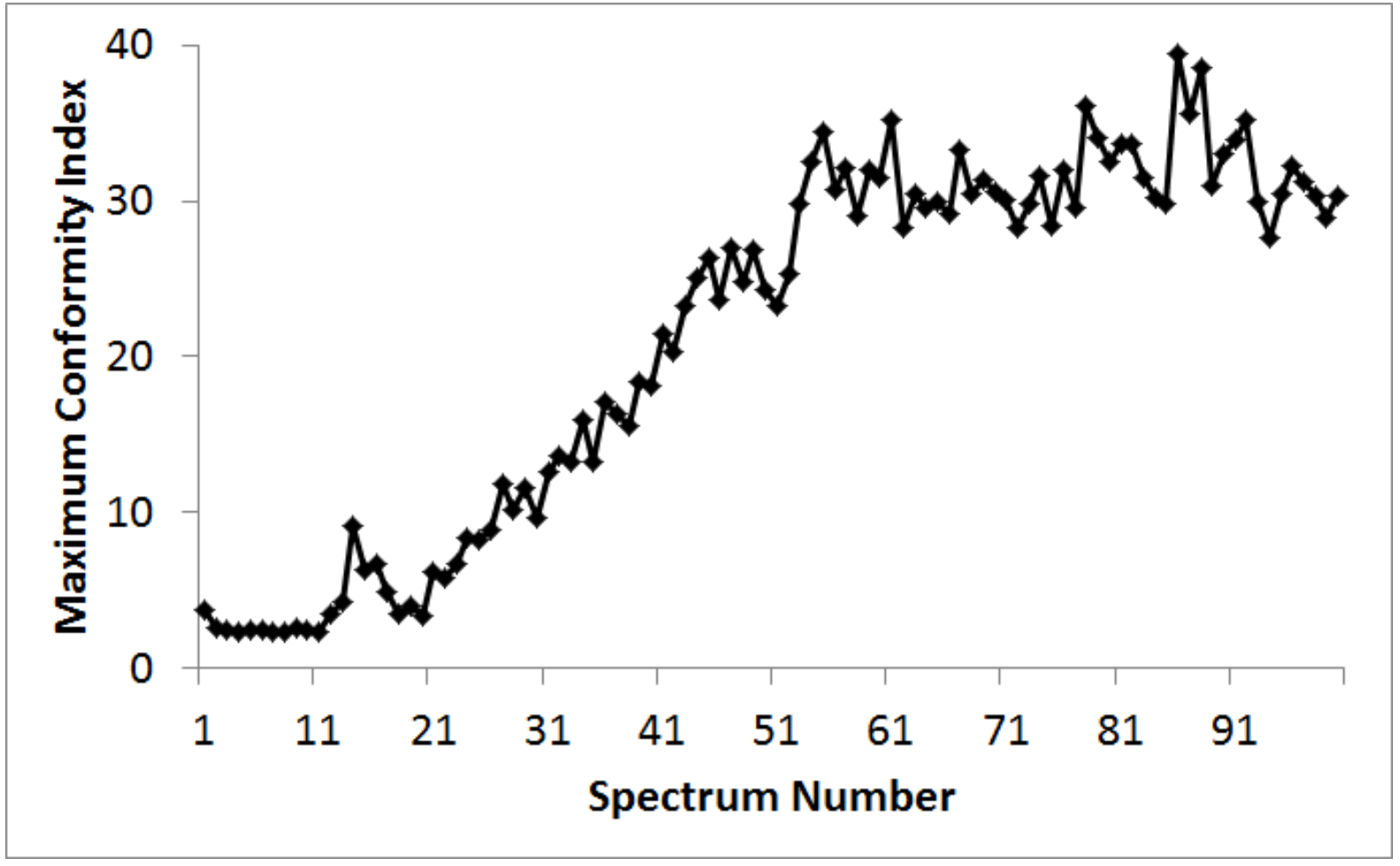

Figure 6. Conformity index of NIR spectra collected during injection moulding of PET containing $0.09 \%$ PLA.
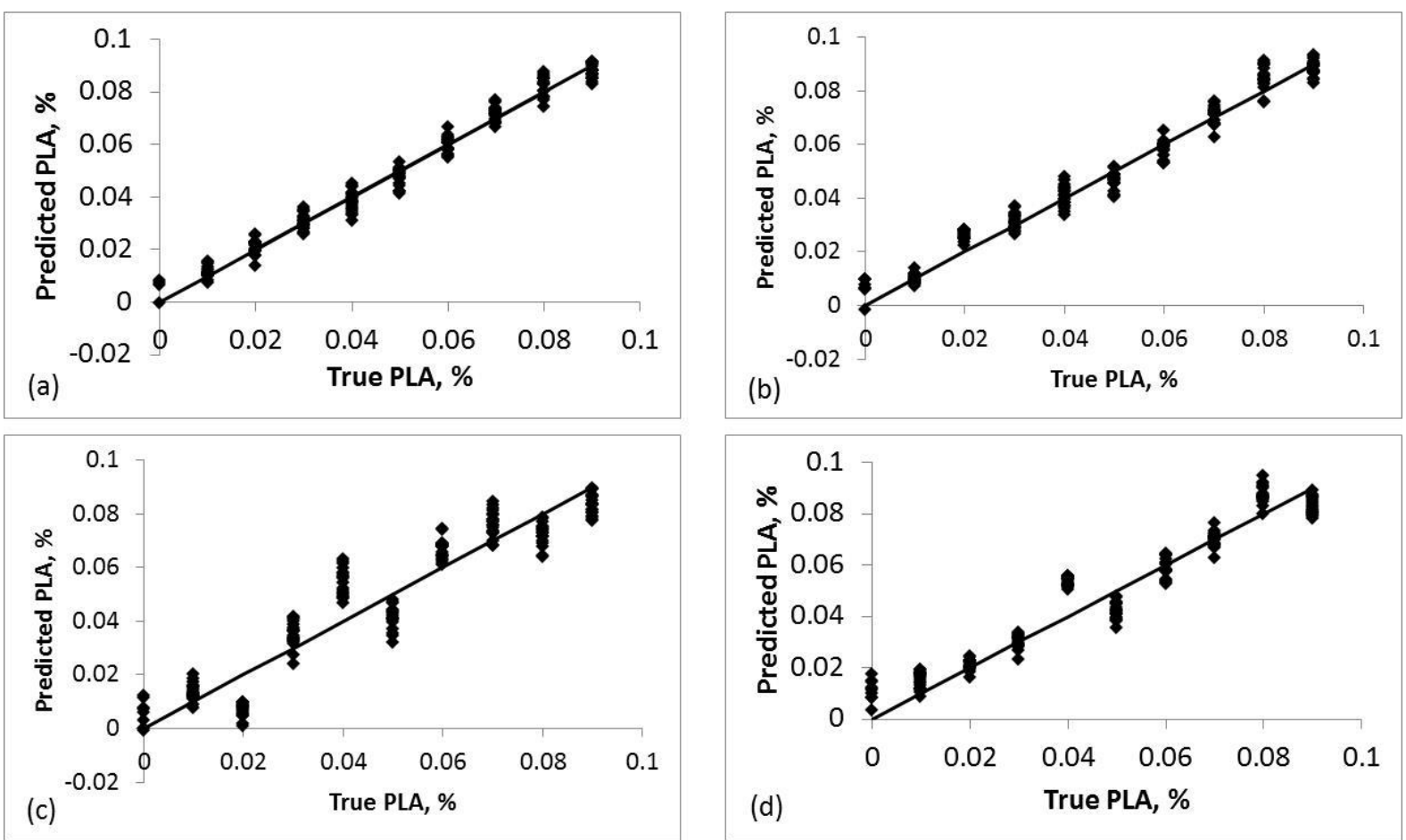
Figure 7. Validation plots (predicted PLA\% vs. true PLA\%) for four chemometric models: (a) A (b) B (c) C (d) E.

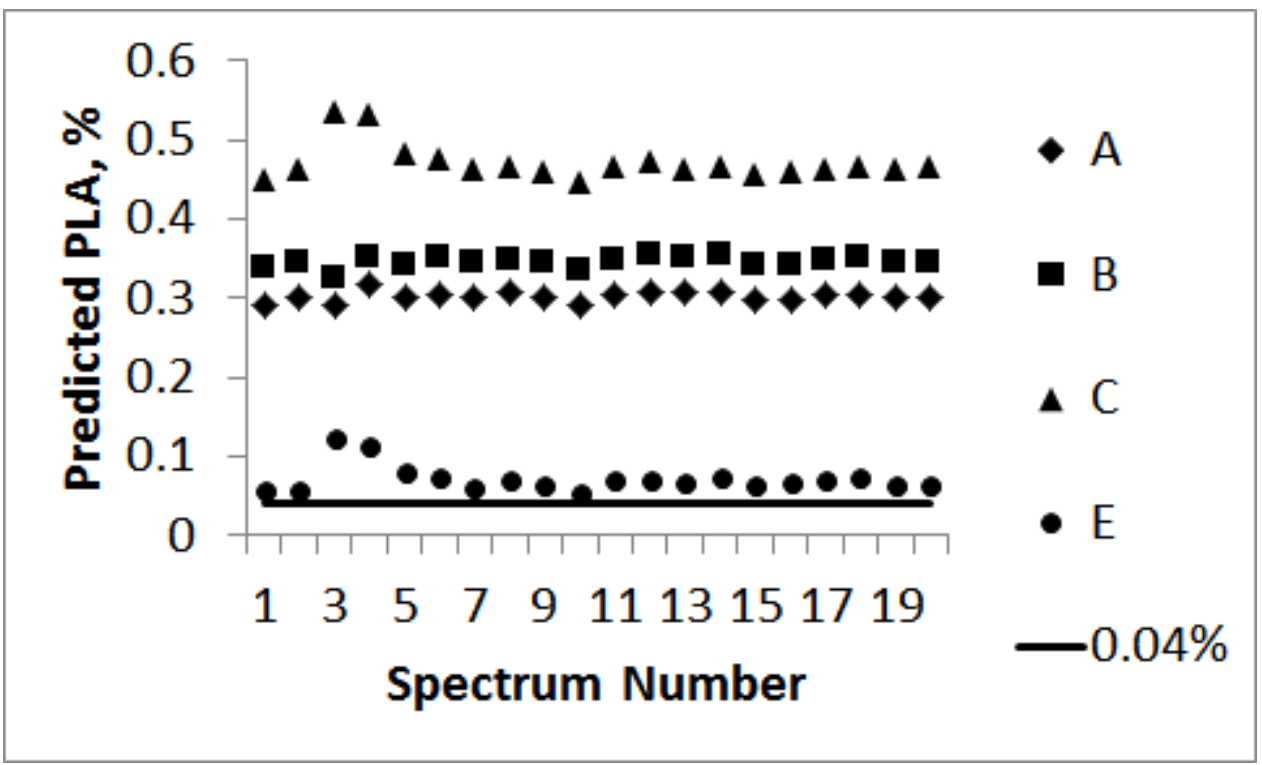

Figure 8. Prediction of PLA concentration in PET polymer melt from near infrared spectra by four chemometric models. 
Table 1. Injection moulding operating conditions for collection of in-line near-infrared spectra.

\begin{tabular}{ll}
\hline Parameter & Value \\
\hline Nozzle & $265^{\circ} \mathrm{C}$ \\
Zone 1 & $265^{\circ} \mathrm{C}$ \\
Zone 2 & $260^{\circ} \mathrm{C}$ \\
Zone 3 & $260^{\circ} \mathrm{C}$ \\
Tool & $25^{\circ} \mathrm{C}$ \\
Injection Pressure & $480 \mathrm{bar}$ \\
Holding Pressure & $300 \mathrm{bar}$ \\
Holding Time & $4 \mathrm{sec}$ \\
Injection & $30-40 \mathrm{ccm} / \mathrm{s}$ \\
Shot Size & $10 \mathrm{ccm}$
\end{tabular}

Table 2. Spectral regions selected for optimisation algorithms.

\begin{tabular}{cc}
\hline $\begin{array}{c}\text { Default spectral regions } \\
\text { used for NIR } \\
\text { optimisation algorithm } \\
\left(\mathrm{cm}^{-1}\right)\end{array}$ & $\begin{array}{c}\text { Spectral regions specific to } \\
\text { PLA selected for GenB } \\
\text { optimisation algorithm } \\
\left(\mathrm{cm}^{-1}\right)\end{array}$ \\
\hline $1200-7500$ & $9000-8240$ \\
$7500-6100$ & $7500-7000$ \\
$6100-5450$ & $6710-5900$ \\
$5450-4805$ & $5900-5750$ \\
$4805-4250$ & $5750-5600$ \\
& $5600-5400$ \\
& $5400-5000$ \\
& $5000-4650$
\end{tabular}


Table 3. Parameters of optimised PLS models for chemometric measurement of PLA in PET.

\begin{tabular}{|c|c|c|c|c|c|c|c|}
\hline Model & $\begin{array}{l}\text { Spectral } \\
\text { Region } \\
\text { Selection }\end{array}$ & $\begin{array}{l}\text { Algo- } \\
\text { rithm }\end{array}$ & $\begin{array}{l}\text { Spectral } \\
\text { Preproc. }\end{array}$ & Rank & RMSECV & $r^{2}$ & $\begin{array}{c}\text { Spectral } \\
\text { Regions } \\
\left(\mathrm{cm}^{-1}\right)\end{array}$ \\
\hline \multirow{3}{*}{ A } & & & & & & & $9750-7500$ \\
\hline & Default & NIR & None & 5 & 0.0035 & 98.2 & $5450-4600$ \\
\hline & & & & & & & $9000-8240$ \\
\hline \multirow[t]{3}{*}{ B } & PLA specific & GenB & None & 6 & 0,0044 & 974 & $6711-5900$ \\
\hline & 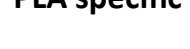 & & & & & & $5400-4650$ \\
\hline & & & & & & & $6711-5900$ \\
\hline \multirow[t]{2}{*}{ C } & PLA specific & GenB & None & 7 & 0.0094 & 88.3 & $5400-4650$ \\
\hline & & & & & & & $9000-8240$ \\
\hline \multirow{2}{*}{ D } & PI A cnerific & Gen R & $1^{\text {st }}$ Deriv & 9 & 2014 & 700 & $6711-5900$ \\
\hline & PLA SpecITIC & Gens & 1 Deriv & 9 & 0.0142 & 10.0 & $5400-4650$ \\
\hline E & PLA specific & GenB & $1^{\text {st }}$ Deriv. & 6 & 0.0074 & 92.9 & $9000-8240$ \\
\hline \multirow[t]{2}{*}{$\mathbf{F}$} & PLA specific & GenB & $1^{\text {st }}$ Deriv. & 9 & 0.0134 & 76.4 & $9000-8240$ \\
\hline & & & & & & & $5400-4650$ \\
\hline
\end{tabular}

